\section{TRAINING THE WHOLE DENTAL IMPLANT TEAM}

Dentsply Friadent offers a variety of education options for every member of the dental implant team. Many of the most popular courses feature live surgery and hands-on sessions to help develop practical skills and lectures by leading experts provide theoretical teaching in implant surgery and prosthetics.

During 2012, there are more comprehensive year courses for those who want to be able to place dental implants for their own patients. For those who are already active in dental implantology, the 2012 programme offers additional surgical courses to help clinicians gain the skills to tackle a wider range of cases.

Combined with appropriate formal tuition, one-to-one mentoring is an essential component of training in implant dentistry. More than 30 accomplished implantologists listed on courses4implants.com are keen to share their wealth of knowledge to help colleagues get involved with implant treatment.

Dentists who wish to restore implant cases can quickly learn to do so through an even more extensive selection of short courses and training events.

Dental nurses and hygienists can gain the knowledge and confidence to support implant dentists on courses around the UK. Practical interactive training is also available for dental technicians who wish to undertake implant work for dental surgeons.

As a complement to attendance on traditional courses, dental professionals can gain knowledge in this field with the click of a mouse. Implantology postgraduate education is more widely available than ever before via the online learning resources. In addition, practice development seminars help dentists to treat more patients and grow their business. Reader response number 57

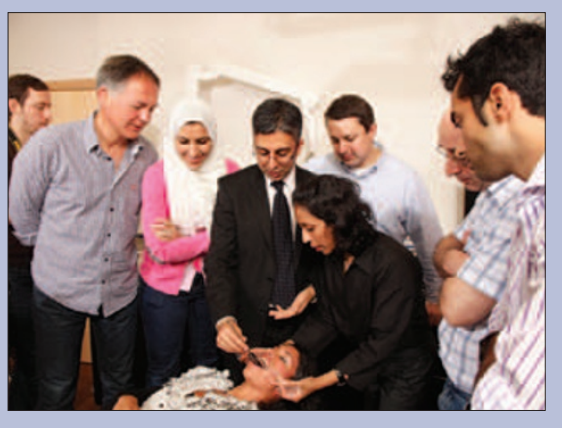

\section{OPTIMAL IMAGE CONTRAST AND DENSITY}

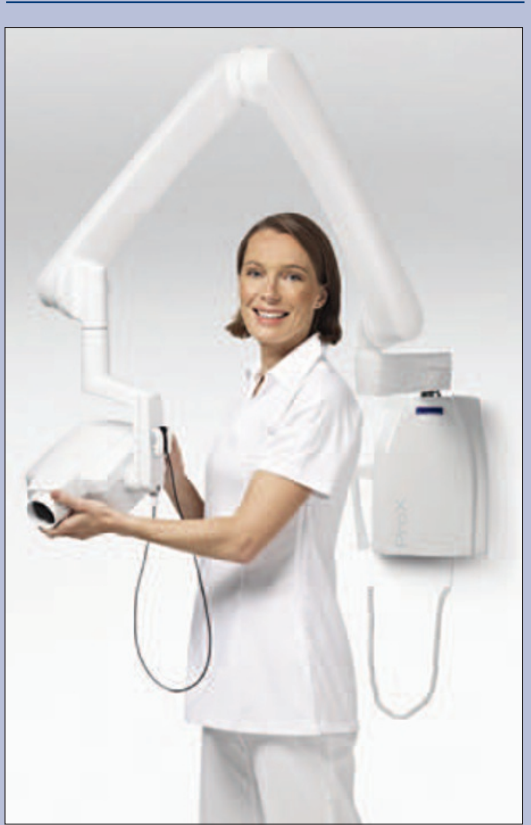

The Finnish dental equipment manufacturer Planmeca Oy has expanded its collection of imaging products with a new intraoral X-ray unit - Planmeca ProX. The advanced unit provides easy and precise positioning, a straightforward procedure and high-quality, highresolution images.

The Planmeca ProX intraoral X-ray unit offers an optimal image contrast and density for every diagnostic need and anatomical condition. This is enabled by variable kilovolts (50-70 kV) and milliamperes (2-8 $\mathrm{mA}$ ). The freely selectable exposure parameters maximise the diagnostic value of intraoral radiography. The focal spot size of the X-ray tube is 0.4 $\mathrm{mm}$, ensuring an optimal resolution and clear images. The unit is pre-programmed with 66 quick settings for different exposure value combinations. The imaging parameters are selected from the intuitive control panel.

The unique design of the X-ray tube head makes aiming exceptionally easy. The steady $\mathrm{X}$-ray unit arm provides smooth and precise movements, ensuring driftfree and accurate positioning. Reader response number 59 operation. The device heats modelling tools and wax much more rapidly owing to its low thermal mass construction. Most users of the improvise some means of catching wax drips. With the introduction of has incorporated a built-in drip tray featuring a removable stainless much cleaner to use. The SafeAir Heatstream is ideal for domiciliary visits; having fewer exposed hot surfaces and being able to cool to packing temperature much faster, it does not require carrying gas canisters or inflammable liquids in vehicles when visiting patients outside the clinic. The handy storage and transport case makes visits even easier.

The SafeAir - Heatstream is available from most major dental suppliers and direct from Carbolite.

Reader response number 58 